\title{
Chronic Myeloid Leukemia: How to Overcome the Tyrosine Kinase Inhibitors Resistance
}

\author{
Yinzhe GE and Mang XIAO* \\ Department of Otolaryngology Head and Neck Surgery, China \\ *Corresponding author: Mang Xiao, Department of Otolaryngology Head and Neck Surgery, China
}

\section{ARTICLE INFO}

Received: 幽 May 15, 2019

Published: May 21, 2019

Citation: Yinzhe GE, Mang XIAO. Chronic Myeloid Leukemia: How to Overcome the Tyrosine Kinase Inhibitors Resistance. Biomed J Sci \& Tech Res 18(2)2019. BJSTR. MS.ID.003118.

\begin{abstract}
Chronic Myeloid Leukemia (CML) is a cancer stem cell-based hematopoietic malignancy that is characterized by unregulated myeloid cell proliferation in the bone marrow and peripheral blood. Tyrosine kinase inhibitors (TKIs) has been the front-line therapy for CML by effectively inhibiting the BCR-ABL oncoprotein and achieved durable clinical responses in many patients. However, resistance to TKI frequently occurs and poses a major obstacle for the treatment of CML. Recent studies have drawn a more complete picture of TKI resistance that it involves multiple aspects of CML cells, including deregulated signaling, BCR-ABL mutations, as well as the intrinsic insensitivity of CML stem cells. Here, we review the history of CML, current therapy challenges and solutions.
\end{abstract}

Abbreviations: CML: Chronic Myeloid Leukemia; TKIs: Tyrosine kinase inhibitors; MPD: Myeloproliferative Disease; IFN $\alpha$ : Interferon- $\alpha$; BMT: Bone Marrow Transplantation; BCR: Breakpoint Cluster Region Gene; ABL: Abelson Kinase Gene; SH2: Src-Homology-2; IM: Imatinib; Ph Chromosome: Philadelphia Chromosome

\section{Introduction}

The first description of Chronic Myeloid Leukemia (CML) dates back to 1840s, when David Craigie and his colleges described a few cases of patients with fever, splenomegaly and leukocytosis, and subsequent death [1]. In the 1960s, Nowell and Hungerford, and later by others, noticed a consistent feature, the presence of a minute chromosome--Philadelphia chromosome (Ph chromosome), from the blood of CML patients [2]. Ph chromosome was further characterized by Rowley to be originated from a reciprocal chromosomal translocation event, designated t $(9 ; 22)$ [3]. The major outcome of this chromosomal translocation is the production of an oncogenic fusion protein, BCR-ABL, and the causal relationship of BCR-ABL and CML was further established by Daley et al by showing induction of CML-like leukemia in mice by virally transducing BCR-ABL gene into murine stem cells [4].

\section{Pathology, Epidemiology and Conventional Therapy}

CML is a myeloproliferative disease (MPD), characterized by elevated levels of white blood cell count, with the majority of cells being mature neutrophils, myelocytes, basophils and eosinophils, usually with few blasts [5]. The uncontrolled leukemia cell accumulation in the peripheral blood and bone marrow, and patients finally succumb to disease with subsequent infiltration of leukemic cells into lung and liver [4]. CML has a median onset age at 53 with the annual incidence of about one to two cases per 100,000 people [6]. CML patients are usually identified in chronic, accelerated and blast crisis phases [5]. Chronic phase patients usually have 4-5 years of latency, and majority of the cells in peripheral blood are neutrophils. BCR-ABL signaling promotes genomic instability and accumulation of additional mutations in CML cells, leading to arrest of CML cells at immature blast cell stage and disease progression. Some CML patients may go through an accelerated phase of about 6-9 months, while other patients may directly enter terminal blast crisis phase [7].

The survival of patients with blast crisis CML patients is often between 3 to 6 months5. In the early days, CML patients were predominantly treated with radiotherapy or chemotherapies with busulfan and hydroxyurea, but neither of them was able to eradicate $\mathrm{Ph}+$ cells effectively [1]. Interferon- $\alpha$ (IFN $\alpha$ ) was introduced in the 1980s to treat CML patients in chronic phase and gradually considered the major option for newly diagnosed CML patients. 
Till now, allogeneic bone marrow transplantation (BMT) is the only proven cure for CML.

\section{BCR-ABL}

$\mathrm{Ph}$ Chromosome is found to be in $>95 \%$ of CML patients, it produces a constitutively active oncogenic tyrosine kinase, BCR$\mathrm{ABL}$, by joining the breakpoint cluster region gene (BCR) with the Abelson kinase gene (ABL or ABL1) [8]. Depending on the fusion junction, BCR-ABL has three isoforms, p190, p210, and p230. Majority of CML patients possess p210 BCR-ABL. This fusion event prevents the shuttling of BCR and ABL gene between cytoplasm and nucleus. The highly conserved Src-Homology-2 (SH2) binding domain in BCR promotes assembly of signaling complexes, leading to constitutive activation of ABL kinase in the cytoplasm and subsequent cell transformation and CML development, bypassing the requirement of various cytokine-stimulated signaling pathways for normal growth and differentiation of hematopoietic cells [9]. The key signaling pathways activated by BCR-ABL include, but not are limited to, RAF/MEK/ERK, JAK/STAT, and PI3K/AKT pathway [10].

\section{Tyrosine Kinase Inhibitor (TKI) and IM Resistance}

The first line TKI, imatinib (IM, also known as Gleevec), was developed in the 1990s. IM inhibits BCR-ABL kinase activity by competing with ATP for the access to the ATP-binding pocket, thus locking BCR-ABL at the inactive conformation and preventing substrate phosphorylation and activation of downstream signaling pathways [11]. IM shows great potency in suppressing disease progression in chronic phase CML patients, however, it is much less effective in patients with advanced diseases, especially in blast crisis patients [12]. These unresponsive events were collectively known as IM resistance [13]. IM resistance stems from the repertoire of secondary mutations in CML cell population, either BCR-ABL kinase domain mutation, BCR-ABL gene amplification or other BCR-ABL independent mutations which confer survival advantages to those cells conceiving them upon IM treatment, therefore selectively resulting in the clonal evolution of this subpopulation and disease progression of CML $[14,15]$. Approximately, 50\% of IM-resistant cases are due to either BCR-ABL kinase domain mutation or BCRABL gene amplification [16,17]. With BCR-ABL mutation, the survival signal is still provided by the constitutive kinase activity of BCR-ABL. However, in the remaining 50\% of resistant cases, BCRABL is wildtype and able to be effectively inhibited by IM, unknown genetic alterations shifted the dependence of CML cells on BCR-ABL for survival.

\section{BCR-ABL Dependent TKI Resistance}

Therapeutic outcome of IM is often compromised by either BCRABL mutations or BCR-ABL gene amplification [18]. Up to now, over 100 mutations have been found to be relevant to TKI resistance, most of which are within the BCR-ABL kinase domain $[19,20]$. Since these mutant leukemic cells are still relying on BCR-ABL activity for survival, it is thus called "BCR-ABL-dependent TKI resistance" [21]. More potent TKIs, such as the second-generation inhibitors, represented by Nilotinib and Dasatinib, and the third-generation inhibitor, represented by Ponatinib, have been developed to recognize and inhibit a variety of BCR-ABL mutants. In contrast to IM, which is discovered through drug screening, Nilotinib was developed based on rational drug design [22]. Nilotinib blocks the catalytic activity of BCR-ABL by binding to the inactive conformation of ABL kinase domain through lipophilic and weak van der wall interactions [23]. Nilotinib has 10-30 folds of increased potency compared to IM in inhibiting BCR-ABL activity and proliferation of BCR-ABL+ cells [24].

While Nilotinib could inhibit a wide range of BCR-ABL mutations, it has no effect on BCR-ABL with T315I mutation. This ineffectiveness might be attributed to the steric hindrance between the 2-methylphenyl phenyl group of Nilotinib and the isoleucinemethyl group of BCR-ABL [23]. Different from most TKIs binding to the inactive conformation of BCR-ABL, Dasatinib binds to the active conformation of ABL kinase with reduced selectivity and less stringent conformational requirement [25]. These binding characteristics of Dasatinib result in a $\sim 325$-fold increased potency against wildtype BCR-ABL than IM and effectively inhibit the majority of BCR-ABL mutations except for T315I [26]. In addition, Dasatinib is also highly active against SRC family kinases including FRG, FYN, HCK, LCK, LYN, and YES, additional tyrosine kinase targets include KIT and PDGFR [27]. CML patients will switch to the second-generation inhibitor, Nilotinib or Dasatinib, when the disease becomes refractory to IM due to resistant mutations [28-29]. Many of these mutations could be inhibited by second-generation TKIs, however, long-term disease remission is not always achieved with sequential TKI treatment. Patients often relapse due to acquired mutations at additional amino acid positions, such as the notorious gatekeeper mutation, T315I $[30,31]$. The third-generation inhibitor, Ponatinib, a dual ABL/SRC inhibitor, is the first TKI demonstrating potent inhibition of T315I mutation and most of known BCR-ABL mutations. This could be explained by the linear structure of ponatinib which helps it avoid steric hindrance with the hydrophobic gatekeeper residues. It is noteworthy that T315I is not the only mutation causing resistance to Nilotinib or Dasatinib. Moreover, the amino acid substitution at 315 position from Threonine to Methionine abolishes the effect of Ponatinib, which was originally designed to target T315I mutation [32]. In addition to these resistant mutations caused by single amino acid substitution, acquirement of mutations at the second or third amino acid positions in the same BCR-ABL protein molecule, dubbed "compound mutations", is another major cause of TKI resistance and disease relapse [32,33].

\section{BCR-ABL Independent TKI Resistance}

The causes of primary resistance to TKI are much more diverse, and sometimes associated with an individual' genetic background. One of the well-known driving mechanism of IM resistance is the overexpression of an SRC family kinase, Lyn, discovered by treating an IM sensitive K562 cell line with increasing dose of 
IM and validated in primary resistant CML samples [34]. As well, overexpression of the MDR1 gene, which encodes p-glycoprotein, a multidrug exporter, mediates imatinib resistance [35]. A recent study carried out a genome-wide shRNA screen in human CML cells and revealed that dozens of genes, upon knockdown, lead to IM resistance. It was shown that this resistance is at least partially caused by transcriptional upregulation of PRKCH, and subsequent activation of downstream MAPK pathway [36].

Oncogene addiction is proposed as the underlying mechanism by which cancer cell can be effectively targeted. It is thought that BCR-ABL inhibition triggers rapid loss of pro-survival signals while gaining pro-apoptotic signals, the highly skewed signaling finally leads to cell death [37]. Nonetheless, more and more studies have shown that cell signaling often responds in a network fashion, and transient activation of compensatory pathways could slow down or circumvent cell death program [38], leading to TKI resistance. One example is the oncogene BCL6, which is suppressed by JAK/STAT pathway. TKI treatment inhibits BCR-ABL and its downstream JAK/ STAT pathway, thus releasing the suppression on BCL6, which then acts in a feedback loop to compromise the efficacy of TKI [39].

\section{TKI Insensitivity and Mechanisms of CML Stem Cells}

In spite of the great efficacy of TKI in suppressing CML progression in general, TKI therapy alone barely leads to a cure. Like many other malignancies, CML is also propagated by a small population of stem cells, CML stem cells. Mounting evidence has demonstrated that CML stem cells remain alive even after BCR-ABL activity has been effectively inhibited by TKIs [40-42], indicating that CML stem cells are not depending on BCR-ABL itself for survival. Additionally, IM discontinuation often leads to CML relapses and this relapse does not result from BCR-ABL mutation $[43,44]$ again suggesting the long-term persistence of CML stem cells.

It was first noted by Holyoke and colleagues that IM treatment fails to kill all Lin-CD34+ cells isolated from peripheral blood of chronic phase CML patient. Jiang and colleagues further demonstrated that purified CD34+CD38- population exhibited significantly lower sensitivity to IM treatment irrespective of the presence of growth factors [42]. Most recently, Druker and colleagues for the first time showed that IM treatment robustly inhibits tyrosine phosphorylation of BCR-ABL as well as phosphorylation of its downstream substrate CRKL and STAT5 in purified CD34+CD38- cell population, and also confirmed CML stem cell viability could not be affected by IM in the presence or absence of growth factors [45].

Studies in primary CML patient samples and CML mouse models have revealed the essential signaling pathways involved in CML stem cell maintenance. For example, Wnt/ $\beta$-catenin pathway was reported to be essential for the self-renewal and differentiation of leukemic stem cells other than normal HSCs [46]. Global gene profiling of mouse HSCs and CML stem cells (Lin-Sca1+Kit+GFP+) treated with or without IM identified Alox5 as a major CML stem cell maintenance gene [47]. PRKCH/MAPK pathway has also been demonstrated to contribute to IM insensitivity of CML stem cells [36]. More recently, systemic analysis of gene expression at both RNA and protein level in CML stem cells revealed deregulated of p53 and c-Myc signaling network [48]. Using small molecules to increase p53 stability while simultaneously abolishing of c-Myc signaling dramatically enhanced elimination of CML stem cells. Additional reported signaling pathways contributing to IM resistance of CML stem cells includes hedgehog [49] TGF-FOXO [50], BCL6 [51] and HIF1 $\alpha$ [52].

\section{Conclusion}

Taken together, understanding the mechanisms of various types of IM-resistance will provide better opportunities to devise therapeutic strategies for effective treatment of CML and eventually achieve a cure.

\section{Funding Sources}

This study is sponsored by grants from Zhejiang Provincial Natural Science Foundation of China (grant No. LY15H160028). Science and Technology Department of Zhejiang Province (grant No.2019336033)

\section{Declaration of Interests}

All authors have read and approved to submit it to your journal and declare no conflict of interest. None of the contents of this manuscript has been previously published or is under consideration elsewhere. All the authors read and approved the final version of the manuscript prior to submission.

\section{References}

1. Goldman JM (2010) Chronic myeloid leukemia: a historical perspective. Seminars in hematology 47(4): 302-311.

2. Nowell PC, D Hungerford, P Nowell (1960) A minute chromosome in human chronic granulocytic leukemia. Science, 1497

3. Rowley JD (1973) A new consistent chromosomal abnormality in chronic myelogenous leukaemia identified by quinacrine fluorescence and Giemsa staining. Nature 243: 290-293.

4. Daley GQ Van Etten RA, Baltimore D (1990) Induction of chronic myelogenous leukemia in mice by the $\mathrm{P} 210 \mathrm{bcr} / \mathrm{abl}$ gene of the Philadelphia chromosome. Science 247(4944): 824-830.

5. Faderl, S, Talpaz M, Estrov Z, O Brien S, Kurzrock R, et al. (1999) The biology of chronic myeloid leukemia. The New England journal of medicine 341(3): 164-172.

6. Redaelli A, Bell C, Casagrande J, Stephens J, Botteman M, et al. (2004) Clinical and epidemiologic burden of chronic myelogenous leukemia. Expert review of anticancer therapy 4(1): 85-96.

7. Schiffer CA, Hehlmann R, Larson R (2003) Perspectives on the treatment of chronic phase and advanced phase CML and Philadelphia chromosome positive ALL (1). Leukemia 17(4): 691-699.

8. Kurzrock R, Shtalrid M, Romero P, Kloetzer WS, Talpas M, et al. (1987) A novel c-abl protein product in Philadelphia-positive acute lymphoblastic leukaemia. Nature 325(6105): 631-635.

9. Ren R (2005) Mechanisms of BCR-ABL in the pathogenesis of chronic myelogenous leukaemia. Nature reviews Cancer 5(3): 172-183. 
10. Steelman LS, Pohnert SC, Shelton JG, Franklin RA, Bertrand FE, et al (2004) JAK/STAT, Raf/MEK/ERK, PI3K/Akt and BCR-ABL in cell cycle progression and leukemogenesis. Leukemia 18(2): 189-218.

11. Schindler T, Bornmann W, Pellicena P, Miller WT, Clarkson B, et al. (2000) Structural mechanism for STI-571 inhibition of abelson tyrosine kinase. Science 289(5486): 1938-1942.

12. Sawyers CL, Hochhaus A, Feldman E, Goldman JM, Miller CB, et al. (2002) Imatinib induces hematologic and cytogenetic responses in patients with chronic myelogenous leukemia in myeloid blast crisis: results of a phase II study. Blood 99(10): 3530-3539.

13. Shah NP (2005) Loss of response to imatinib: mechanisms and management. Hematology / the Education Program of the American Society of Hematology. American Society of Hematology. Education Program pp. 183-187.

14. Kurzrock R, Kantarjian HM, Druker BJ, Talpaz M (2003) Philadelphia chromosome-positive leukemias: from basic mechanisms to molecular therapeutics. Ann Intern Med 138(10): 819-830.

15. Savona M, Talpaz M (2008) Getting to the stem of chronic myeloid leukaemia. Nature reviews Cancer 8(5): 341-350.

16. Kantarjian HM, Giles F, Quintas Cardama A, Cortes J (2007) Important therapeutic targets in chronic myelogenous leukemia. Clin Cancer Res 13(4): 1089-1097.

17. Wu J, Meng F, Kong LY, Peng Z, Ying Y, et al. (2008) Association between imatinib-resistant BCR-ABL mutation-negative leukemia and persistent activation of LYN kinase. J Natl Cancer Inst 100(13): 926-939.

18. Gorre ME, Mohammed M, Ellwood K, Hsu N, Paquette R, et al. (2001) Clinical resistance to STI-571 cancer therapy caused by BCR-ABL gene mutation or amplification. Science 293(5531): 876-880.

19. Soverini S, Hochhaus A, Nicolini FE, Gruber F, Lange T, et al. (2011) BCR-ABL kinase domain mutation analysis in chronic myeloid leukemia patients treated with tyrosine kinase inhibitors: recommendations from an expert panel on behalf of European LeukemiaNet. Blood 118(5): $1208-1215$.

20. (2007) Laboratory WMRG Guidelines for mutation analysis of BCR/ABL kinase domain: Interpreting TKI-Resistance Mutations in CML Patients. WMRGL.

21. Bhamidipati PK, Kantarjian H, Cortes J, Cornelison AM, Jabbour E (2013) Management of imatinib-resistant patients with chronic myeloid leukemia. Therapeutic advances in hematology 4(2): 103-117.

22. Kantarjian H, Giles F, Wunderle L, Bhalla K, O Brien S, et al. (2006) Nilotinib in imatinib-resistant CML and Philadelphia chromosomepositive ALL. The New England journal of medicine 354(24): 2542-2551.

23. Manley PW, Cowan Jacob SW, Mestan J (2005) Advances in the structural biology, design and clinical development of Bcr-Abl kinase inhibitors for the treatment of chronic myeloid leukaemia. Biochimica et biophysica acta $1754(1-2): 3-13$.

24. Kantarjian HM, Giles F, Gattermann N, Bhalla K, Alimena G, et al. (2007) Nilotinib (formerly AMN107), a highly selective BCR-ABL tyrosine kinase inhibitor, is effective in patients with Philadelphia chromosomepositive chronic myelogenous leukemia in chronic phase following imatinib resistance and intolerance. Blood 110(10): 3540-3546.

25. Eck MJ, Manley PW (2009) The interplay of structural information and functional studies in kinase drug design: insights from BCR-Abl. Current opinion in cell biology 21(2): 288-295.

26. Olivieri A, Manzione L (2007) Dasatinib: a new step in molecular target therapy. Annals of oncology: official journal of the European Society for Medical Oncology / ESMO 18 (6): vi42-46.

27. Schittenhelm MM, Shiraga S, Schroeder A, Corbin AS, Griffith D, et al (2006) Dasatinib (BMS-354825), a dual SRC/ABL kinase inhibitor inhibits the kinase activity of wild-type, juxtamembrane, and activation loop mutant KIT isoforms associated with human malignancies. Cancer research 66(1): 473-481.
28. Weisberg E, Manley PW, Cowan Jacob SW, Hochhaus A, Griffin JD (2007) Second generation inhibitors of BCR-ABL for the treatment of imatinibresistant chronic myeloid leukaemia. Nature reviews Cancer 7(5): 345356.

29. Cortes J, Jabbour E, Kantarjian H, Yin CC, Shan J, et al. (2007) Dynamics of BCR-ABL kinase domain mutations in chronic myeloid leukemia after sequential treatment with multiple tyrosine kinase inhibitors. Blood 110(12): 4005-4011.

30. Cortes JE, Hagop Kantarjian, Neil P Shah, Dale Bixby, Michael J Mauro, et al. (2012) Ponatinib in refractory Philadelphia chromosome-positive leukemias. The New England journal of medicine 367: 2075-2088.

31. O Hare T, Shakespeare WC, Zhu X, Eide CA, Rivera VM, et al. (2009) AP24534, a pan-BCR-ABL inhibitor for chronic myeloid leukemia, potently inhibits the T315I mutant and overcomes mutation-based resistance. Cancer cell 16(5): 401-412.

32. Zabriskie MS, Eide CA, Tantravahi SK, Vellore NA, Estrada J, et al. (2014) BCR-ABL1 compound mutations combining key kinase domain positions confer clinical resistance to ponatinib in $\mathrm{Ph}$ chromosome-positive leukemia. Cancer cell 26(3): 428-442.

33. Shah NP, Skaggs BJ, Branford S, Hughes TP, Nicoll JM, et al. (2007) Sequential ABL kinase inhibitor therapy selects for compound drugresistant BCR-ABL mutations with altered oncogenic potency. The Journal of clinical investigation 117(9): 2562-2569.

34. Donato NJ, Wu JY, Stapley J, Gallick G, Lin H, et al. (2003) BCR-ABL independence and LYN kinase overexpression in chronic myelogenous leukemia cells selected for resistance to STI571. Blood 101(2): 690-698.

35. Illmer T, Schaich M, Platzbecker U, Freiberg Richter J, Oelschlägel U, et al. (2004) P-glycoprotein-mediated drug efflux is a resistance mechanism of chronic myelogenous leukemia cells to treatment with imatinib mesylate. Leukemia 18(3): 401-408.

36. Ma L, Shan Y, Bai R, Xue L, Eide CA, et al. (2014) A therapeutically targetable mechanism of BCR-ABL-independent imatinib resistance in chronic myeloid leukemia. Science translational medicine 6(252): 252ra121.

37. Sharma SV, Gajowniczek P, Way IP, Lee DY, Jiang J, et al. (2006) A common signaling cascade may underlie "addiction" to the Src, BCR-ABL, and EGF receptor oncogenes. Cancer cell 10(5): 425-435.

38. Logue JS, Morrison DK (2012) Complexity in the signaling network: insights from the use of targeted inhibitors in cancer therapy. Genes \& development 26(7): 641-650.

39. Duy C, Hurtz C, Shojaee S, Cerchietti L, Geng H, et al. (2011) BCL6 enables $\mathrm{Ph}+$ acute lymphoblastic leukaemia cells to survive BCR-ABL1 kinase inhibition. Nature 473(7347): 384-388.

40. Graham SM, Jørgensen HG, Allan E, Pearson C, Alcorn MJ, et al. (2002) Primitive, quiescent, Philadelphia-positive stem cells from patients with chronic myeloid leukemia are insensitive to STI571 in vitro. Blood 99(1): 319-325.

41. Hamilton A, Helgason GV, Schemionek M, Zhang B, Myssina S, et al (2012) Chronic myeloid leukemia stem cells are not dependent on BcrAbl kinase activity for their survival. Blood 119(6): 1501-1510.

42. Jiang X, Zhao Y, Smith C, Gasparetto M, Turhan A, et al. (2007) Chronic myeloid leukemia stem cells possess multiple unique features of resistance to BCR-ABL targeted therapies. Leukemia 21(5): 926-935.

43. Yhim HY, Lee NR, Song EK, Yim CY, Jeon SY, et al. (2012) Imatinib mesylate discontinuation in patients with chronic myeloid leukemia who have received front-line imatinib mesylate therapy and achieved complete molecular response. Leukemia research 36(6): 689-693.

44. Ross DM, Susan Branford, John F Seymour, Anthony P Schwarer, Christopher Arthur, et al. (2013) Safety and efficacy of imatinib cessation for CML patients with stable undetectable minimal residual disease: results from the TWISTER study. Blood 122: 515-522.

45. Corbin AS, Agarwal A, Loriaux M, Cortes J, Deininger MW, et al. (2011) Human chronic myeloid leukemia stem cells are insensitive to 
imatinib despite inhibition of BCR-ABL activity. The Journal of clinica investigation 121(1): 396-409.

46. Zhao C, Blum J, Chen A, Kwon HY, Jung SH, et al. (2007) Loss of betacatenin impairs the renewal of normal and CML stem cells in vivo. Cancer cell 12(6): 528-541.

47. Chen Y, Hu Y, Zhang H, Peng C, Li S (2009) Loss of the Alox5 gene impairs leukemia stem cells and prevents chronic myeloid leukemia. Nature genetics 41(7): 783-792.

48. Abraham SA, Hopcroft LE, Carrick E, Drotar ME, Dunn K, et al. (2016) Dual targeting of p53 and c-MYC selectively eliminates leukaemic stem cells. Nature 534(7607): 341-346.

49. Zhao C, Chen A, Jamieson CH, Fereshteh M, Abrahamsson A, et al. (2009) Hedgehog signalling is essential for maintenance of cancer stem cells in myeloid leukaemia. Nature 458(7239): 776-779.

\section{ISSN: 2574-1241}

DOI: 10.26717/BJSTR.2019.18.003118

Mang Xiao. Biomed J Sci \& Tech Res

(C) This work is licensed under Creative

Submission Link: https://biomedres.us/submit-manuscript.php
50. Naka K, Hoshii T, Muraguchi T, Tadokoro Y, Ooshio T, et al. (2010) TGFbeta-FOXO signalling maintains leukaemia-initiating cells in chronic myeloid leukaemia. Nature 463(7281): 676-680.

51. Hurtz C, Hatzi K, Cerchietti L, Braig M, Park E, et al. (2011) BCL6mediated repression of p53 is critical for leukemia stem cell survival in chronic myeloid leukemia. The Journal of experimental medicine 208(11): 2163-2174.

52. Wang Y, Liu Y, Malek SN, Zheng P, Liu Y (2011) Targeting HIF1alpha eliminates cancer stem cells in hematological malignancies. Cell stem cell 8(4): 399-411.

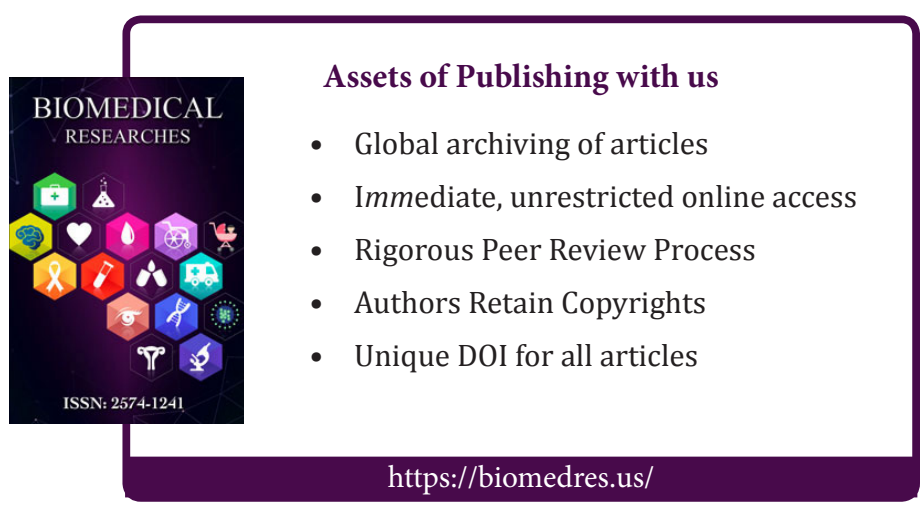

